\title{
Données et échantillons liés à la santé pour la recherche
}

La loi relative à la recherche sur l'être humain (LRH) permet, à certaines conditions, d'établir un consentement général (CG), par lequel toute personne peut accepter d'une manière générale l'utilisation de ses données et échantillons dans le cadre de projets de recherche ultérieurs. Certains hôpitaux suisses ont d'ores et déjà introduit le CG avec toutefois des formulaires d'information et de consentement divergents, compliquant ainsi la collaboration à des projets de recherche au-delà des institutions. L'Académie Suisse des Sciences Médicales (ASSM) et l'organisation faîtière des commissions d'éthique suisses pour la recherche sur l'être humain (swissethics) ont élaboré ensemble un modèle de CG Suisse. Celui-ci comprend une information succincte (avec consentement) et une brochure d'information détaillée.

Le modèle a été conçu par un groupe de travail'; parallèlement, la Swiss Biobanking Platform (SBP) a mis à disposition des guidelines, dans lesquelles la gestion des données et des échantillons est standardisée. ${ }^{2}$ De même, des représentants des organisations de patients, des hôpitaux et de la recherche ont participé à l'élaboration du modèle.

\section{Ci-après un aperçu des trois éléments fondamentaux}

1. L'option du consentement au prélèvement d'un échantillon pour la biobanque

Le modèle de CG couvre l'utilisation de données et d'échantillons génétiques et non génétiques codés provenant d'examens diagnostiques et de traitements de routine. La possibilité d'obtenir le consentement à des prélèvements supplémentaires de sang, de salive ou d'urine à des fins de recherche a également été intégrée au modèle de CG.
2. Données non génétiques codées: le consentement au lieu de l'opposition

La LRH (art. 33 al. 2) autorise la réutilisation de données de santé non génétiques codées sans consentement, dans la mesure où le donneur ne s'y est pas opposé. Le modèle de CG Suisse va au-delà de la LRH en exigeant également un consentement pour l'utilisation de données de santé non génétiques codées.

3. Attitude à adopter face aux révocations et aux résultats fortuits

Le modèle de CG Suisse part du principe qu'en cas de révocation, les données et les échantillons ne peuvent plus être utilisés à des fins de recherche. Concernant l'attitude à adopter en cas de résultats fortuits, le modèle laisse une marge d'appréciation. En règle générale, plus la maladie (existante ou susceptible de se développer) est grave, plus les constatations concrètes sont fiables, et plus on dispose d'une option thérapeutique ou d'une mesure de prévention, plus la personne concernée doit être informée rapidement.

\section{Procédure de consultation sur le site web de l'ASSM}

Le projet de modèle de CG Suisse est soumis à une consultation publique jusqu'au 31 mars 2017. Les institutions et personnes intéressées sont invitées à s'exprimer: assm.ch/consultation-2017-03

La version définitive du modèle de CG Suisse sera approuvée par swissethics et publiée en avril 2017. Des modèles spécifiques aux enfants et adolescents et à leurs parents seront élaborés prochainement. 